\title{
Controversial \\ Entrapment \\ Neuropathies
}

\author{
William W. Campbell, MD, COL, MC, USA ${ }^{a}$, \\ Mark E. Landau, MD, LTC, MC, USA ${ }^{\text {b, * }}$
}

\section{KEYWORDS}

- Thoracic outlet syndrome - Radial tunnel syndrome

- Arcade of Struthers - Piriformis syndrome

- Tarsal tunnel syndrome

There is no significant disagreement about the major common entrapment neuropathies, such as carpal tunnel syndrome (CTS), ulnar neuropathy at the elbow, and peroneal neuropathy at the knee. There is some minor disagreement about fine points regarding the clinical presentation, electrodiagnosis, and management, but there is certainly no major disagreement, such as whether these entities exist. In contrast, there is a group of entrapment syndromes about which there is major disagreement, including whether or not they even exist. The book entitled Tunnel Syndromes lists some 25 tunnel syndromes, most of which are so obscure that most neurologists have never heard of them, for example, Alcock's tunnel syndrome. ${ }^{1}$ There are other entrapment syndromes about which clinical questions arise on a regular basis, and which are the subject of this discussion. These include thoracic outlet syndrome (TOS), radial tunnel syndrome (RTS), ulnar nerve entrapment at the arcade of Struthers, piriformis syndrome (PS), and tarsal tunnel syndrome (TTS).

\section{THORACIC OUTLET SYNDROME}

TOS has a long and controversial history. It was first described by Kinnier Wilson in approximately 1900 , and there is some reason to think that one of his original patients may have had CTS. TOS was established in the minds of physicians as a cause of acroparesthesias for some 40 odd years before CTS and cervical radiculopathy were described. What we now refer to as CTS was referred to by various names in the early twentieth century, including "tardy median palsy"; it did not receive its name until 1947 in a paper by Lord Brain and colleagues. ${ }^{2}$ In patients who have acroparesthesias, TOS is one of the first possibilities come to mind for many physicians, but for most neurologists, it is nearly the last thing to come to mind. The perception regarding the prevalence of TOS between surgeons and neurologists is at variance on the order of 100:1. First rib resections and other surgical procedures continue to be performed on a regular basis in the United States. There are occasional disasters from these procedures, with severe damage to the brachial plexus. ${ }^{3,4}$ It has been estimated that the likelihood of postsurgical brachial plexopathy is about equal to the likelihood of the diagnosis of TOS being accurate in the first place. Cherington and Cherington ${ }^{5}$ found an interesting correlation between TOS surgery and method of reimbursement in Colorado, with Medicaid patients virtually never receiving TOS surgical procedures.

Compression of the neurovascular structures at the thoracic outlet has been blamed on various structures, including cervical ribs or bands, an elongated $\mathrm{C} 7$ transverse process, the scalenus anterior muscle, the pectoralis minor tendon, and

\footnotetext{
a Department of Neurology, Uniformed Services University of Health Sciences, 4301 Jones Bridge Road, Bethesda, MD 20814, USA

b Department of Neurology, Walter Reed Army Medical Center, 6900 Georgia Avenue, Washington, DC 20307, USA

* Corresponding author.

E-mail address: mark.landau@amedd.army.mil (M.E. Landau).
} 
the humeral head. ${ }^{6,7}$ We should probably recognize five types of TOS: arterial, venous, true neurogenic, disputed, and droopy shoulder syndrome. Vascular TOS occurs most commonly in young athletes, especially those who hyperabduct the shoulder in throwing and in swimming. ${ }^{8}$ In the position of hyperabduction, the humeral head may compress the axillary artery or vein, causing such vascular changes as stenosis, occlusion, poststenotic dilatation, aneurysm formation, and subclavian vein occlusion. ${ }^{9}$

True neurogenic TOS is an entity about which there is substantial agreement. It is rare, attributable to compression of the lower trunk of the plexus by a cervical rib or band. True neurogenic TOS is more common in women and presents with medial arm pain; medial hand and forearm sensory dysfunction; and weakness and atrophy of the thenar, hypothenar, and other intrinsic hand muscles. The thenar eminence, muscles supplied by the median nerve, are typically involved earlier and more severely than the muscles of the hypothenar eminence. The electrodiagnostic abnormalities in true neurogenic TOS include an absent or small medial antebrachial cutaneous amplitude (MABC), followed in decreasing frequency by absent or small median motor, ulnar sensory, and ulnar motor amplitudes. ${ }^{10}$ The MABC nerve conduction study (NCS) abnormalities closely parallel the median motor response changes, leading some investigators to conclude that the damage of neurogenic TOS is in a T1 myotomal distribution (Fig. 1). ${ }^{11}$ Needle electromyography (EMG) shows denervation and chronic neurogenic motor unit potential changes in the distribution of the lower trunk of the plexus. ${ }^{10}$ One typical pattern would include fibrillation potentials and positive sharp waves in the abductor and opponens pollicis brevis muscles, associated with more chronic denervative changes in the adductor digiti minimi, the first dorsal interosseous, the flexor pollicis longus, and, rarely, the extensor indicus proprius.

The proximal location for neurogenic TOS pathologic findings limits an electromyographer's ability to detect subtle demyelinating changes. Considering CTS, nerve conduction measurements are easily recorded for the sensory and motor fibers of the median nerve as they transverse the wrist. This allows detection of mild demyelinating changes in the sensory fibers, with excellent sensitivity and specificity for diagnosis. Analogous studies for any proximal nerve segments are invariably associated with technical limitations. There is a long-standing myth that TOS can cause slowing of the ulnar motor conduction velocity when stimulating at Erb's point and doing

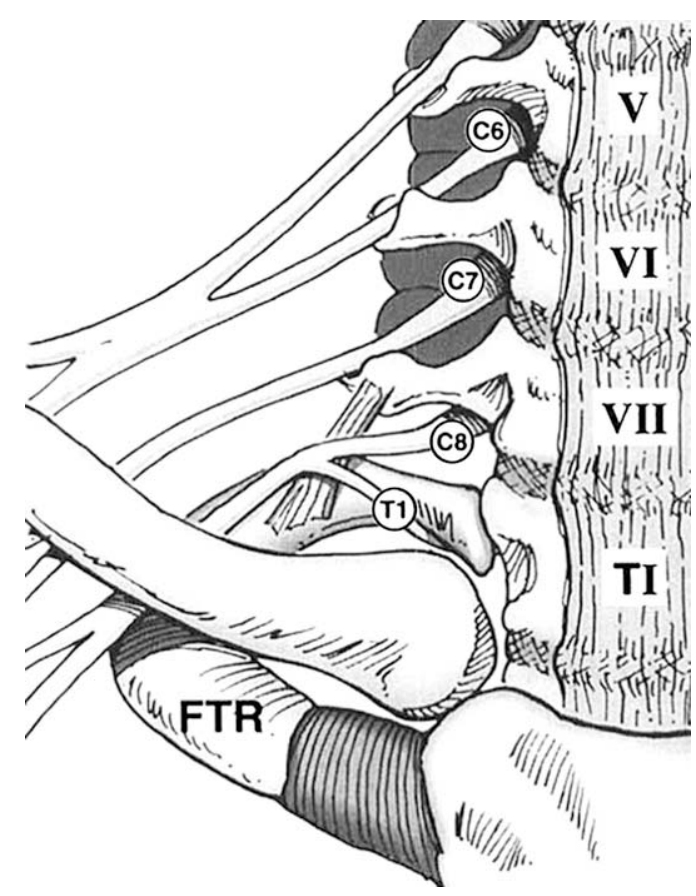

Fig. 1. Neurogenic TOS: ligamentous band from the transverse process of $\mathrm{C} 7$ to the first thoracic rib (FTR) lying in close approximation to the $\mathrm{C} 8$ and T1 anterior primary rami and the proximal portion of the lower trunk. (From Levin KH, Wilbourn AJ, Maggiano HJ. Cervical rib and median sternotomy-related brachial plexopathies: a reassessment. Neurology 1998;50:1412; with permission.)

an "across the plexus" velocity determination. An article was written describing this in the New England Journal of Medicine in $1972 .^{12}$ The article was subsequently proved to be fraudulent research with a fabricated figure and was withdrawn, with a stern editorial rebuke about authorship responsibilities. ${ }^{13}$ This technique has finally gradually fallen into disfavor and is rarely performed currently by reputable electromyographers. Therefore, electrodiagnosis relies on the detectable axonal changes distal to the site of pathologic change resulting from Wallerian degeneration. This supports the argument for those who favor TOS being relatively underdiagnosed in that subtler disease is not detectable by means of objective measures. ${ }^{14}$

Patients who neither have true vascular nor true neurogenic TOS have been referred to as having "disputed neurogenic" TOS. The leading surgical proponents think that $97 \%$ of patients who have TOS are "neurologic," and disputed neurogenic TOS accounts for most of these. ${ }^{14}$ These patients are said to have aching pain radiating from the scapular region down the arm; it can radiate through the axillary region, the triceps area, and 
the medial forearm. Arm flexion or abduction worsens the pain. There may be paresthesias in the medial forearm and hand. There are no motor manifestations, and the results of electrodiagnostic studies are normal. In other words, the diagnosis relies on sensory signs and symptoms, which are entirely subjective. One of the major criteria for the diagnosis of TOS is reported to be a positive Adson maneuver, a physical diagnostic sign that was shown 50 years ago to be positive in $80 \%$ of normal asymptomatic individuals. With the Adson maneuver, the patient's arm hangs at the side, the head is rotated toward the affected side, and the patient is instructed to breathe deeply. The test is considered positive if the radial pulse disappears. In a presentation and abstract at the American Academy of Neurology (AAN) in 2005, some investigators showed that positive "TOS" physical test results can occur in patients who have CTS. ${ }^{15}$ Other provocative maneuvers purportedly abnormal in TOS include the Halstead maneuver, the Wright test, and the Roos overhead exercise test. A positive Halstead test result (exaggerated military position or costoclavicular test) is defined as a decreased radial pulse with the patient standing, with the shoulders far backward and in a downward position so as to narrow the costoclavicular space. A positive Wright test result is also defined as a decreased radial pulse, with the shoulder hyperabducted and the elbow flexed. ${ }^{16}$ A positive Roos or elevated arm stress test (EAST) is reproduction of the patient's symptoms following 3 minutes of rapid opening and closing of the hand, with the arm abducted to $90^{\circ}$ and externally rotated and the elbow flexed to $90^{\circ} .{ }^{17}$ These test results may all be abnormal in healthy subjects. ${ }^{18}$

Recently, neuroimaging has attempted to correlate anatomic variations with clinical symptoms. ${ }^{19-21}$ Thoracic outlet MRI findings have been compared in symptomatic and asymptomatic individuals. Demondion and colleagues ${ }^{20}$ found that patients who had TOS had a smaller costoclavicular distance, a thicker subclavius muscle, and a wider retropectoralis minor space after hyperabduction and external rotation of the arm compared with controls. Nerve compression was noted in $7 \%$ of the symptomatic group and in none of the asymptomatic group. The symptomatic group was defined has having two abnormal provocative test results (Adson maneuver, Wright maneuver, Eden maneuver, Roos test, or Tinel sign in the supraclavicular fossa). Neurologic examinations and electrodiagnostic studies were not performed. In a similar study, Demirbag and colleagues $^{21}$ compared MRI findings of the thoracic outlet in an adducted versus hyperabducted position of patients and controls. This study blinded an examiner in performing provocative tests. Interestingly, two thirds of subjects in the control group had a positive Adson test result. The specificity for the exaggerated military position and hyperabduction test were considerably better, with $8.3 \%$ and $25 \%$, respectively, of controls having a positive test result. All subjects had normal EMG findings, but no details were provided regarding the extent to which imitators, such as CTS or cervical radiculopathy, were excluded. The study concluded that considerably more variation of predefined radiographic parameters was seen in the MRI scans between the neutral and hyperabducted position in the patient group. Less variation of these findings was seen in the control group. Furthermore, in the hyperabducted position, compression of venous $(52.3 \%$ versus $41.7 \%)$, arterial (23.0\% versus $1.4 \%)$, and neural $(12.1 \%$ versus none) segments was more common in the patient groups.

At this time, it is unclear whether a combination of MRI findings and provocative clinical maneuvers can substantiate the diagnosis of disputed neurogenic TOS. Surgical intervention is frequently offered to patients who fail conservative management, but the evidence for efficacy and the perioperative complications vary considerably. A surgeon who confidently supports the existence of disputed TOS reports $90 \%$ success rates, with perioperative complications less than $1 \% .{ }^{14}$ Conversely, another study showed persistent disability in $60 \%$ of patients 1 year after surgery and perioperative complications greater than $30 \% .{ }^{22}$ Complications reported in the literature include postoperative brachial plexopathy, pleural tears, pneumothorax, hemorrhage, and coronary artery spasm.

The most basic of medical principles must be invoked in any discussion of TOS-first, do no harm. Disputed TOS is a sensory syndrome without definitive objective findings to support its existence. The clinical tools used for its diagnosis are exceptionally sensitive, with poor specificity. The surgical treatment can result in objective deficits in a not insignificant percentage of patients, many of whom had a normal thoracic outlet before surgery.

\section{RADIAL TUNNEL SYNDROME}

RTS is a condition in which there is chronic pain in the lateral elbow region, allegedly because of entrapment of elements of the radial nerve by various structures in a tunnel in the region of the supinator muscle. ${ }^{23}$ There is considerable doubt as to whether this clinical entity or the anatomic 
tunnel exists. ${ }^{24,25}$ As with the other controversial entrapments, it is essentially a pain syndrome without objective clinical or electrophysiologic manifestations. There is significant clinical overlap between RTS and lateral epicondylitis (LE). LE, or tennis elbow, is a common condition that results from overuse of the extensor and supinator muscles, typically by playing tennis. It produces pain and tenderness over the region of the lateral epicondyle and usually responds to conservative treatment with rest and anti-inflammatory agents. Examination of the elbow usually shows tenderness over the epicondyle, which is maximal just distal to the epicondyle. With the elbow extended, wrist extension or elbow supination against resistance causes increased pain in the symptomatic area, and forceful wrist flexion or pronation stretches the involved region and also causes pain. Occasional patients fail to respond to conservative therapy and develop chronic lateral elbow pain sometimes referred to as "resistant tennis elbow." There is a school of orthopedic surgeons who believe that this syndrome of resistant tennis elbow is attributable to entrapment of the radial nerve in the radial tunnel. ${ }^{26,27}$

The radial tunnel is a nebulous anatomic passageway; nerve compression has been attributed to no less than eight different anatomic structures here. With anatomic studies, some groups found that a radial tunnel existed in most subjects, whereas other groups, seemingly equally competent, found that the radial tunnel existed in none of their subject cadavers (Fig. 2). There is considerable doubt simply on an anatomic basis as to whether there is such an entity as the radial tunnel. The would-be tunnel is approximately $5 \mathrm{~cm}$ long and located on the anterior aspect of the proximal radius. ${ }^{28,29}$ It originates just proximal to the radial capitulum and terminates distally at the point where the posterior interosseous nerve (PIN) passes deep to the superficial portion of the supinator muscle. The capsule of the radial capitulum forms the floor of the tunnel. The brachioradialis, extensor carpi radialis brevis (ECRB), and longus muscles form the lateral wall, and the biceps tendon and brachialis muscle constitute the medial wall. The anatomic structures implicated in PIN entrapment include a fibrous band linking the brachioradialis to the brachialis, a cluster of blood vessels overlying the nerve (leash of Henry), the medial tendinous edge of the ECRB, the arcade of Frohse, the distal border of the supinator muscle, fibrous bands within the supinator muscle, and the septum between the extensor carpi ulnaris and the extensor digitorum.

The chief symptom of RTS is pain over the lateral aspect of the proximal forearm, with

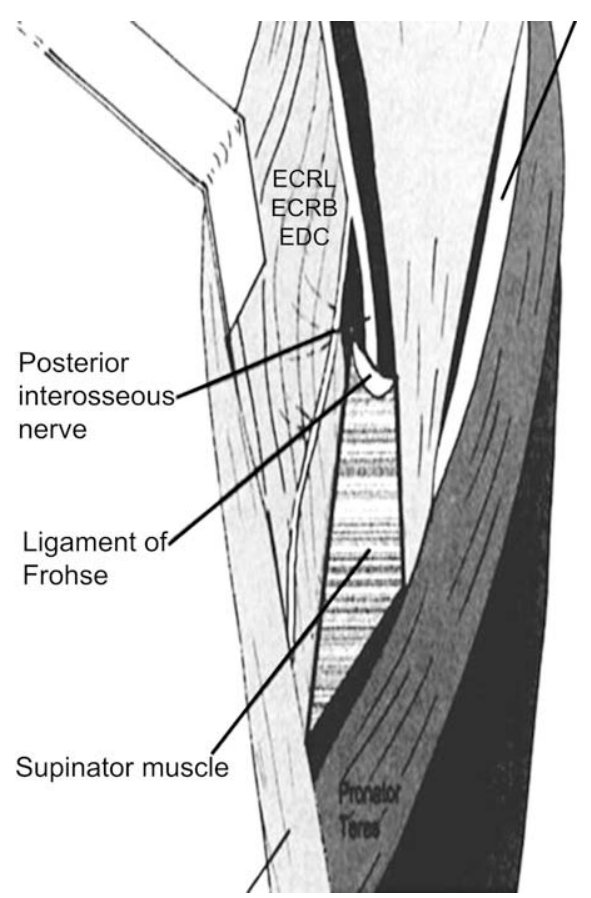

Fig. 2. The radial nerve branches into the posterior interosseous nerve and the superficial radial nerve. The posterior interosseous nerve traverses deep to the ligament of Frohse and the supinator muscle. ECRL and ECRB, extensor carpi radialis longus and brevis; EDC, extensor digitorum communicans. (From Stanley J. Radial tunnel syndrome: a surgeon's perspective. J Hand Ther 2006;19:181; with permission.)

occasional radiation distally or proximally. The pain may be associated with a definitive injury or after excessive use but not invariably. There are none of the typical hallmarks of a PIN lesion, such as finger extensor weakness (finger drop) to a greater degree than wrist extensor weakness. Proponents of RTS have suggested that the PIN provides sensation for joints and muscles and can produce pain when damaged, despite not providing cutaneous sensation. There are only subtle differences between RTS and LE. In RTS, palpation tenderness is demonstrated at the point where the radial nerve enters the supinator muscle, approximately $5 \mathrm{~cm}$ distal to the lateral epicondyle. In LE, the tenderness is directly over the lateral epicondyle, the origin of the extensor muscles, as opposed to the muscles themselves. ${ }^{28}$ In RTS, pain is elicited by fully extending the elbow, followed by resistance against forearm supination. Supposedly, contraction of the supinator increases compression of the arcade of Frohse on the nerve. ${ }^{28}$ Resisting middle finger extension with the forearm pronated and extended may also elicit pain in the supinator region. 
Electrodiagnostic testing is useful in excluding other etiologies of upper extremity pain, because there are no specific findings seen in RTS. Usually, the results of the study are normal, without any evidence of radial or PIN abnormalities. Some investigators have claimed slowing of radial motor nerve conduction velocity with stimulation at the spiral groove while recording over the extensor digitorum communis. ${ }^{23}$

When conservative measures fail, some investigators recommend surgical exploration with "radial tunnel release" in an attempt to relieve the symptoms. ${ }^{30,31}$ The same procedure can also relieve the symptoms of LE, because division of the superficial portion of the supinator muscle also relieves tension on the lateral epicondyle. Posterior interosseous neuropathy may occur as a complication of radial tunnel release.

\section{Ligament of Struthers and Arcade of Struthers Nerve Injuries}

The ligament of Struthers and the arcade of Struthers are two different anatomic structures that are often confused. The ligament of Struthers is a fibrous band that extends from an anomalous supracondylar spur on the humerus down to the medial epicondyle, which may compress the median nerve (Fig. 3). Struthers ${ }^{32}$ described this ligament and has been recognized eponymically for it. Approximately $1 \%$ of the population has this anomaly, but only rarely does it cause nerve injury. ${ }^{33}$ In contrast, the arcade of Struthers consists of filmy, occasionally substantive, tissue along the medial intermuscular septum, which may compress the ulnar nerve (Fig. 4). It is in no way related to the ligament of Struthers. In fact, Struthers did not describe the tissue that later came to be called the arcade of Struthers. ${ }^{34}$ The eponymic designation is erroneous.

As the ulnar nerve descends through the upper arm, it moves from the anterior to the posterior compartment through the intermuscular septum. The so-called "arcade of Struthers" is an aponeurotic strand that extends from the medial intermuscular septum to the medial head of the triceps, about $8 \mathrm{~cm}$ proximal to the medial epicondyle. In one anatomic study, it was seen in $13.5 \%$ of the limbs, but in no instance did it compress the ulnar nerve. ${ }^{35}$

\section{Proximal median mononeuropathy by the ligament of Struthers}

The median nerve, along with the brachial artery, runs beneath the ligament of Struthers, but only rarely does it cause median nerve injury. Plain radiographs reveal the supracondylar spur, but the ligament may be present without a spur.

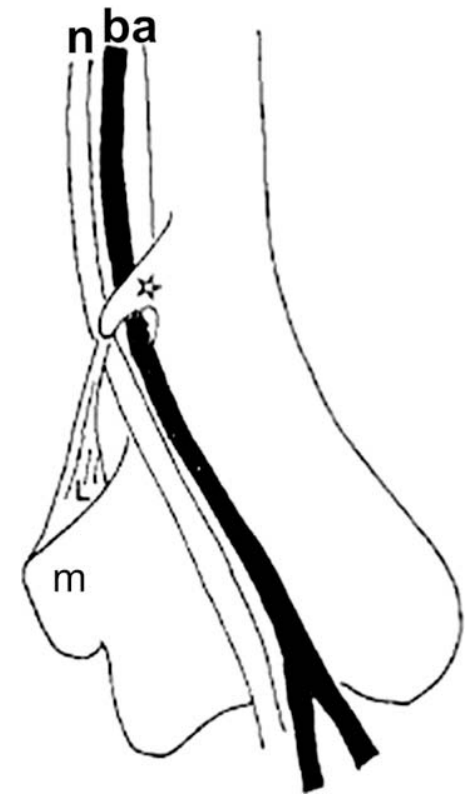

Fig. 3. Ligament of Struthers between the supracondylar spur ( $\left(\mathrm{r}^{2}\right)$ and medial epicondyle $(\mathrm{m})$. Median nerve (n) and brachial artery (ba). (From Aydinlioglu A. Bilateral median nerve compression at the level of Struther's ligament. Case report. J Neurosurg 2000; 92:694; with permission.)

Symptoms include pain above the elbow and local tenderness in addition to neurologic deficits in the distribution of the median nerve. There is not much controversy regarding this entity when there is objective evidence of median neuropathy.

\section{Ulnar mononeuropathy attributable to the arcade of Struthers}

During ulnar transposition surgery, the medial intermuscular septum must be released or the ulnar nerve may become secondarily compressed after it is transposed anteriorly. In such cases re-exploration of the nerve shows deformity and compression of the nerve at the point of entrapment by the inadequately released medial intermuscular septum, and this has been referred to as the arcade of Struthers. This posttransposition syndrome is well recognized and not a subject for debate. ${ }^{36-40}$ The debate concerns whether or not the medial intermuscular septum or an arcade of Struthers can cause a spontaneous compression syndrome of the ulnar nerve. ${ }^{35}$ There is disagreement in the literature about the existence of the arcade of Struthers in a patient who has not had an ulnar transposition. Some anatomic studies have found tissue consistent with an arcade of Struthers in anatomic specimens, whereas other investigators have reported that it virtually never exists. ${ }^{41-44}$ The situation is analogous to that with RTS. It is 


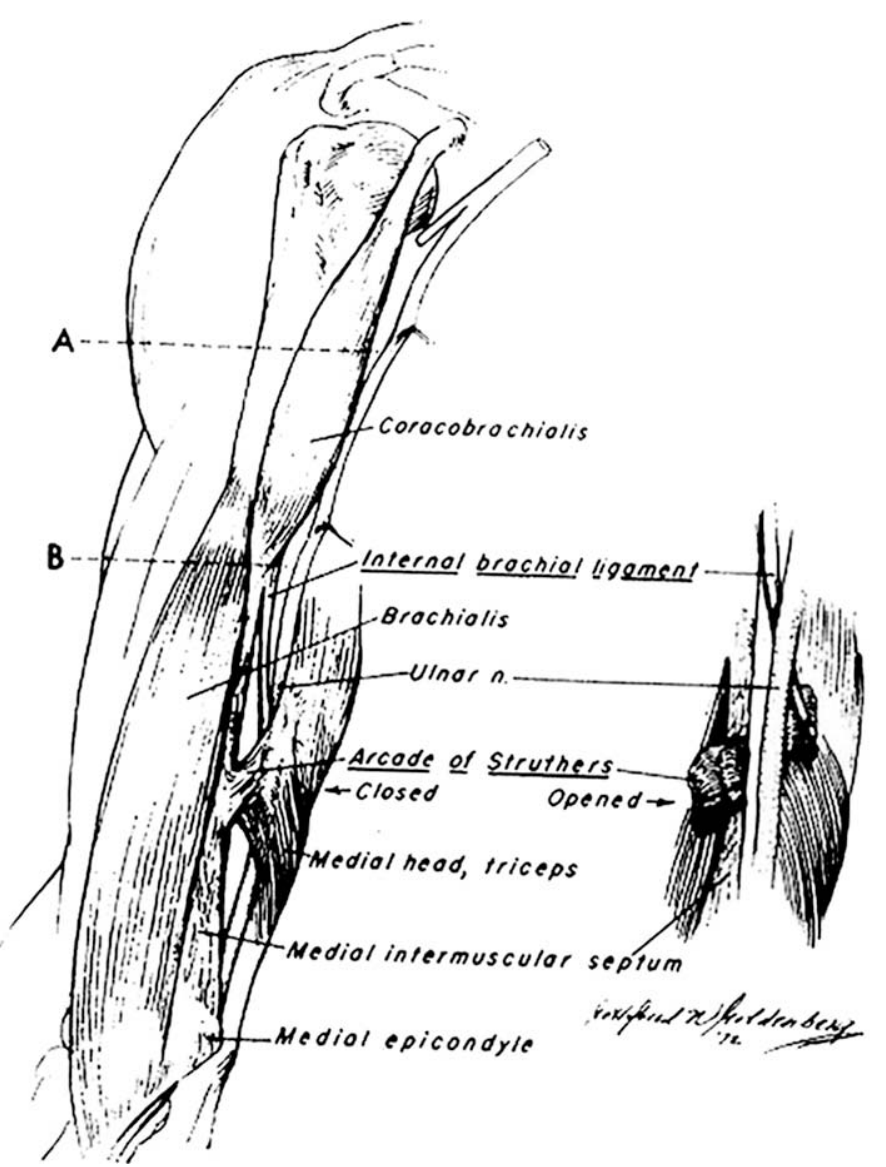

Fig. 4. Arcade of Struthers is located between the medial head of the triceps and the medial intermuscular septum. The ulnar nerve lies deep. (From Kane E, Kaplan EB, Spinner M. Sur le trajet du nerf cubital au niveau du bras [Observations on the course of the ulnar nerve in the arm]. Ann Chir 1973;27:487-96; with permission.) unclear anatomically whether the structure exists. Spinner ${ }^{45}$ suggested that a "friction neuritis" could develop from repeated movement of the nerve against bony irregularities of the distal humerus and that an arcade of Struthers could be a contributing factor. The arcade of Struthers, as a mechanism of ulnar compression in the unoperated arm, is extraordinarily rare or a myth, with some other etiology more likely responsible. The first author of the only three cases reported in peer-reviewed literature is Ochiai. ${ }^{46,47}$ The clinical findings were identical to ulnar mononeuropathy at the elbow, but the electrodiagnostic findings showed slowing of nerve conduction velocity or conduction block 70 to $100 \mathrm{~mm}$ proximal to the medial epicondyle.

\section{PIRIFORMIS SYNDROME}

The PS is a condition allegedly attributable to compression of the sciatic nerve by the piriformis muscle, causing pain in the buttock radiating down the posterior lower extremity, simulating radiculopathy. There is some problem with terminology. The term piriformis syndrome should only be used to designate proximal sciatic neuropathies in which the piriformis muscle itself is involved in the etiology. There are many other causes of proximal sciatic neuropathy, such as gluteal trauma with hematoma, injection palsy, compression, hip arthroplasty, hip fracture, or endometrial implant. ${ }^{48}$ Although the sciatic nerve may be involved near the piriformis in these conditions, none should be legitimately referred to as PS.

The piriformis is one of an array of small muscles that lie deep in the gluteal region: the pectineus, piriformis, obturators, gemelli, and quadratus femoris. These short muscles are probably more important as postural stability muscles for the hip joint rather than as prime movers. The piriformis originates from the anterior sacral surface, passes through the greater sciatic foramen, and inserts onto the medial side of the upper border of the greater trochanter (Fig. 5). The piriformis rotates the extended thigh laterally but acts as an abductor when the hip is flexed. Just below the piriformis lies the gemellus superior. At its exit from the pelvis, the sciatic nerve exists as two discrete fascicles: the peroneal division and the tibial division. 


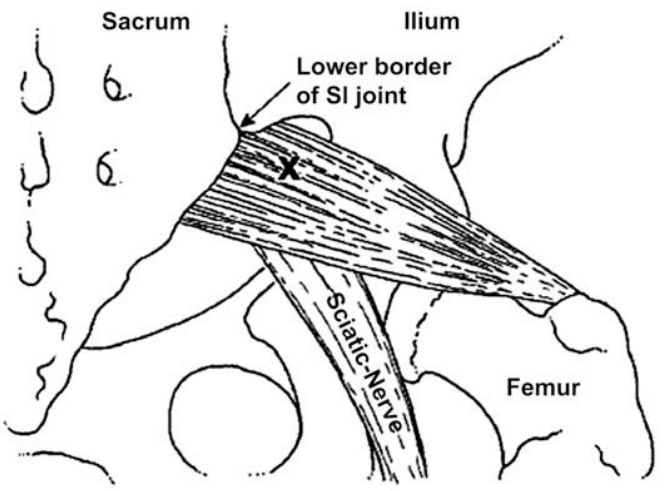

Fig. 5. Piriformis muscle overlying the sciatic nerve. (From Benzon HT. Piriformis Syndrome: Anatomic concsiderations, a New Injection Technique, and a Review of the Literature. Anesthesiology 2003;98:1443; with permission.)

These are most often encased in a common epineural sheath and appear to be a single nerve. The two separate components can easily be dissected apart, however. Anomalies of the exit pattern of the sciatic nerve from the pelvis can occur. ${ }^{49}$ The normal pattern is for the intact sciatic nerve to exit just below the piriformis muscle in the groove between the piriformis and the gemellus superior. In some patients, the sciatic nerve splits: one division exits below the piriformis muscle, and the other exits above it. In some patients, the piriformis is also bifid and one or both elements of the sciatic nerve pierce the muscle. The entire intact nerve may exit directly through the middle of the belly of the piriformis, or one division may exit through the belly of muscle, whereas the other division exits above or below. The incidence of this anomaly varies widely by ethnic origin. In a series of American cadavers, there was some anomaly of sciatic nerve exit in $10 \% .^{50}$ The piriformis is muscle is a small structure, perhaps 3 to $5 \mathrm{~cm}$ wide at its widest point and perhaps $1 \mathrm{~cm}$ thick. The sciatic nerve at that point is a large robust structure.

There are numerous problems with the concept of the PS, and a great debate on how common this entity is. ${ }^{51}$ It is not uncommon to see articles in the literature that stress the uncertainty about the syndrome (eg, "The piriformis syndrome is overdiagnosed" versus "The piriformis syndrome is underdiagnosed" or "The piriformis syndromemyth or reality?") ${ }^{52-54}$ Most estimates of the incidence of PS in patients with back pain estimate its incidence at $1 \%$ to $2 \%$ of cases, but some practitioners claim to see dozens to hundreds of patients per year. One problem with PS is that the nerve is large in relation to the muscle, and it is difficult to see how such a small and relatively insubstantial structure as the piriformis muscle could significantly compress the sciatic nerve. The PS was first described in 1928, long before lumbosacral radiculopathy was recognized as a clinical entity, and long before radiologic imaging or electrodiagnosis existed. Discogenic pain, arising from the annulus itself, without a radicular component, can radiate as far as the knee, causing radiating pain in the buttock and leg but without objective evidence of radiculopathy. Pain in the buttock and leg when sitting is one of the classic symptoms of PS but is also quite common in radiculopathy. Many, perhaps most, patients who have lumbosacral radiculopathy attributable to disc herniation have maximal pain in the sitting position.

The pathophysiologic mechanism of sciatic involvement in PS is unclear. Some investigators believe the presence of an anatomic anomaly is irrelevant, ${ }^{53}$ although others contend that PS requires some exit anomaly. ${ }^{55}$ Nobody has described an abnormal leg movement, such as the external rotation that would accompany a contraction of the piriformis, as part of the syndrome. Therefore the "spasm" must be isometric, without muscle shortening, and it is therefore difficult to envision how nerve compression could occur. It would make just as much sense to claim that spasm of the gemellus superior pressing up against the nerve could cause the nerve compression symptoms. The PS is virtually the only compression or entrapment neuropathy attributable to a muscle. Virtually every other one is attributable to compression by a fascial band, aponeurosis, ligament, or other such rigid structure (eg, transverse carpal ligament, humeroulnar arcade, arcade of Frohse, sublimis bridge, lancinate ligament). Nevertheless, the proponents of PS believe that one of the largest and most robust nerves in the body is being compressed by a soft and compliant structure, which is extremely atypical in the class of disorders that we ordinarily include as entrapment neuropathies.

There are two clinical scenarios that need to be considered. One is the patient with physical examination and electrodiagnostic evidence of proximal sciatic neuropathy. Can the mechanism be PS in the absence of other etiologies of nerve damage? The other clinical scenario regards patients who have buttock pain or buttock pain with radiation down the posterior leg; no other typical signs or symptoms of nerve injury, including paresthesias, hypesthesias, hyporeflexia, weakness or atrophy; and a normal electrodiagnostic assessment. What is the likelihood that a chronic or chronic recurrent compression of the sciatic nerve could account for the pain without the other neurologic manifestations? There is little definitive evidence 
in the literature to support the diagnosis of PS in either case. Even its proponents refer to it as a "loose cluster of symptoms." 56 The clinical criteria include pain in the buttock and usually some part of the course of the sciatic nerve distal to it, tenderness in the region of the intersection of the piriformis muscle and the sciatic nerve, and positive straight leg raise at $15^{\circ}$ less than on the unaffected side, or less than $60^{\circ}$ when PS is bilateral. None of these have been critically evaluated, and are certainly not specific. The piriformis muscle is small and lies deep beneath the massive gluteus maximus; yet, proponents claim to be able to palpate piriformis spasm in patients who have the syndrome ("the princess and the pea sign"). Some of the physical maneuvers allegedly indicative of the syndrome are contradictory. The Pace test consists of sciatic pain produced by abduction of the hip against resistance, presumably causing the muscle to contract against the nerve, reproducing the symptoms. ${ }^{57}$ Yet another maneuver, the Freiburg test, reproduces the pain with forced internal rotation, stretching the muscle. ${ }^{58}$ It would seem the Freiburg test should, if anything, decompress the nerve by stretching the muscle, removing the pressure, and improving the symptoms. How these two diametrically opposite maneuvers could reproduce the pain is difficult to reconcile. In the Beatty maneuver, the patient lies painful side up with the leg flexed and the hip abducted, holding the knee on the painful side several inches off the table; this allegedly reproduces the pain. ${ }^{59}$ There have been no validation studies of any of these tests.

Electrophysiologic criteria have been proposed to assist in diagnosis of PS. ${ }^{56,60}$ In the flexed, adducted, and internally rotated (FAIR) test, the posterior tibial $\mathrm{H}$ reflex is measured with the patient prone and legs in the neutral position, and then again with the hip flexed, adducted, and internally rotated. A latency prolongation of greater than 3 SDs is stated to have greater than $80 \%$ sensitivity and specificity. There are multiple limitations, however, to performing $\mathrm{H}$ reflexes. One is that they are not routinely obtainable in patients older than the age of 60 years. ${ }^{61}$ Second, a prolongation of latency is not specific for the location of the abnormality, because lesions at the nerve root or plexus could also cause prolongations. Whether or not lesions at these locations can also become apparent only in the postures of the FAIR test has not been systematically evaluated. One needs to question the validity of the test as performed by the proponents of the test. The mean age of the patients in their two studies was older than 50 years, yet they did not describe one case in which the $\mathrm{H}$ reflex could not be found. ${ }^{56,60}$ Furthermore, they claimed an ability to attain $\mathrm{H}$ waves in greater than 300 subjects for the peroneal nerve; yet, it is universally accepted by electrodiagnosticians that peroneal $\mathrm{H}$ waves are rarely obtainable. Finally, the waveforms depicted in their studies were biphasic with a negative initial deflection, whereas most $\mathrm{H}$ waves are triphasic with an initial positive deflection.

Treatments recommended for PS include physical therapy, local injections, botulinum toxin injections, and surgery. We are somewhat placated by the $94 \%$ of 918 patients referred to physicians who strongly believe in this entity, getting conservative therapy only, without surgical interventions. ${ }^{60}$

\section{TARSAL TUNNEL SYNDROME}

The TTS is attributable to compression of the tibial nerve within a fibro-osseous passageway behind the medial malleolus. There is widespread agreement regarding the existence of the syndrome, but there are differences of opinion regarding its epidemiology as an etiology for foot pain and paresthesias, particularly in diabetics.

The roof of the tarsal tunnel is the flexor retinaculum (lancinate ligament) and the tendinous arch of the abductor hallucis $(\mathrm{AH})$. The lancinate ligament is thin and insubstantial compared with the transverse carpal ligament. The posterior tibial nerve passes under the ligament and then divides into larger medial and smaller lateral plantar branches. In $7 \%$ of individuals, the tibial nerve branches before the tarsal tunnel. ${ }^{62}$ The medial plantar branch innervates the $\mathrm{AH}$, flexor digitorum brevis, flexor hallucis brevis, and the first lumbrical, and it provides sensation to the medial part of the sole of the foot and the medial three toes. The lateral plantar branch innervates the rest of the deep muscles of the foot and provides sensation to the lateral part of the sole of the foot and the lateral two toes. The calcaneal branch provides sensation to the heel; it usually arises proximal to the tarsal tunnel, but the origin is variable.

The common symptoms of TTS are pain in the area of the medial malleolus with occasional radiation into the medial aspect of the foot. To be convinced of nerve involvement, there should be paresthesias or dysesthesias on the plantar aspect of the foot. Although intrinsic foot muscles may become weak or atrophic, these manifestations rarely interfere with function and go overlooked by patients. The objective physical findings in TTS are few. Weakness of the foot muscles cannot be demonstrated on neurologic examination. There may be hypesthesia over the sole, especially in the medial plantar distribution, usually with sparing of the heel (calcaneal innervation). 
There may be tenderness behind the medial malleolus and a Tinel's sign over the tarsal tunnel. A stress test similar to the Phalen test for CTS has been described. The ankle is passively maximally everted and dorsiflexed, the toes are pulled up, and this position is held for 5 to 10 seconds to reproduce the symptoms. ${ }^{63}$

The differential diagnosis includes arthritis, plantar fasciitis, plantar callosities, vascular disease, or small fiber sensory neuropathies. The diagnosis is often made to explain foot pain of no other apparent origin, even in absence of any neurologic deficit. The causes of TTS include external compression by ill-fitting footwear or casts, ankle trauma, thickened flexor retinaculum, ganglia, posttraumatic fibrosis, and others. ${ }^{64}$ The plantar nerves may be injured distal to the tarsal tunnel from local trauma, compression by tendon sheath cysts, or AH hypertrophy. ${ }^{64}$

There are electrodiagnostic findings that can support the clinical diagnosis of TTS. Ideally, the most sensitive test would detect conduction slowing of sensory nerve fibers as they traverse the tarsal tunnel. This, however, is rarely seen. In most cases of TTS, the medial and lateral plantar sensory nerve action potential (SNAPs) are absent. ${ }^{65}$ This abnormality is not specific for TTS because it may be a manifestation of sensory polyneuropathies or even seen in asymptomatic individuals, particularly those older than the age of 50 years. Prolongation of the medial and lateral plantar motor latencies has been noted in approximately $20 \%$ to $50 \%$ of individuals with TTS. ${ }^{65,66}$ No published electrodiagnostic studies include needle EMG in the evaluation of TTS. ${ }^{67}$ There are many in the electrodiagnostic community who believe that findings of denervation in the intrinsic foot muscles are common in asymptomatic individuals; therefore, any needle electrode findings would be of little use. ${ }^{68,69}$ Others, however, have disputed this claim. ${ }^{70}$ A pattern of EMG abnormalities that would support the diagnosis includes denervation of intrinsic foot muscles isolated to the symptomatic limb of patients who have unilateral disease, with sparing of abnormalities of the extensor digitorum brevis (EDB). The EDB is the only non-tibial-innervated intrinsic foot muscle. The electrodiagnostic tests are particularly useful for ruling out other neurologic etiologies of foot pain, such as sensory polyneuropathies and radiculopathies.

When conservative measures fail to limit symptomatology, surgical intervention may be necessary. A review of published articles showed symptomatic improvement in $91 \%$ of patients surgically treated. ${ }^{71}$ This is a commonly performed procedure, especially by podiatrists and orthopedic foot surgeons. ${ }^{72}$
Surgical decompression of the tarsal tunnel in isolation or along with other peripheral nerves has been used as an alternative approach to diabetic neuropathy. ${ }^{73-78}$ The hypothesis that underlies this approach is that patients who have polyneuropathy may have increased susceptibility to compression mononeuropathies at potential sites of entrapment. Thus, peripheral nerve surgery for diabetics has also included decompression and neurolysis of the common peroneal nerve at the fibular head and the deep peroneal nerve in the anterior tarsal region. ${ }^{73}$ Note that the sural nerve, which provides sensation to the lateral foot, and the saphenous nerve, which provides sensation to the medial lower leg, were not included. It is not clear how surgery could help sensation or other parameters in these regions of diabetics. Dellon and his coworkers ${ }^{74-78}$ have been the major proponents of this intervention. $\mathrm{He}$ and others claim that nerve decompressions in diabetics improve proprioception, cutaneous sensation, balance, and neuropathic pain and prevent ulcerations.

The AAN issued a practice advisory regarding decompression surgery for the treatment of diabetic neuropathy. ${ }^{79,80}$ Per this systematic review, "the current evidence supporting the utility of decompressive surgery for the treatment of diabetic neuropathy is of poor quality and design." All available studies for this intervention were categorized as class IV studies, and the AAN concluded that this treatment alternative be considered unproved. There was one study that was originally categorized as class III because it was prospective with a blinded evaluator. ${ }^{77}$ It was ultimately downgraded to class IV because it lacked detail regarding electrodiagnostic studies and lacked clarity segregating patients into polyneuropathy versus compression categories. Additionally, scales used to define improvement were deemed "arbitrary." In this study, $79 \%$ of surgically decompressed nerves showed improvement in the twopoint discrimination test. All other studies were nonblind case series using a variety of outcome measures. The panel recommended prospective randomized controlled trials with standardized outcome measures to determine the value of this intervention.

\section{REFERENCES}

1. Pecina MM, Krmpotic-Nemanic J, Markiewitz AD. Tunnel syndromes-peripheral nerve compression syndromes. 3rd edition. Boca Raton (FL): CRC Press LLC; 2001. 
2. Brain WR, Wright AD, Wilkinson M. Spontaneous compression of both median nerves in the carpal tunnel. Lancet 1947;1:277-82.

3. Wilbourn AJ. Thoracic outlet syndrome surgery causing severe brachial plexopathy. Muscle Nerve 1988;11:66-74.

4. Cherington M, Happer I, Machanic B, et al. Surgery for thoracic outlet syndrome may be hazardous to your health. Muscle Nerve 1986;9:632-4.

5. Cherington M, Cherington C. Thoracic outlet syndrome: reimbursement patterns and patient profiles. Neurology 1992;42:943-5.

6. Liu JE, Tahmoush AJ, Roos DB, et al. Shoulder-arm pain from cervical bands and scalene muscle anomalies. J Neurol Sci 1995;128:175-80.

7. Korkmaz N, Ozcakar L. Long cervical costae articulating with the first ribs: a salient case of thoracic outlet syndrome. Am J Phys Med Rehabil 2006;85(1): 104.

8. Katirji B, Hardy RW Jr. Classic neurogenic thoracic outlet syndrome in a competitive swimmer: a true scalenus anticus syndrome. Muscle Nerve 1995; 18:229-33.

9. Rigatelli G, Rinuncini M, Roncon L, et al. Images in cardiovascular medicine. Functional subclavian artery compression caused by thoracic outlet syndrome. Circulation 2005;112(17):e280-1.

10. Aminoff MJ, Olney RK, Parry GJ, et al. Relative utility of different electrophysiologic techniques in the evaluation of brachial plexopathies. Neurology 1988;38:546-50.

11. Levin $\mathrm{KH}$, Wilbourn AJ, Maggiano HJ. Cervical rib and median sternotomy-related brachial plexopathies: a reassessment. Neurology 1998;50(5): 1407-13.

12. Urschel HC Jr, Razzuk MA. Management of the thoracic-outlet syndrome. N Engl J Med 1972;286: 1140-3.

13. Wilbourn AJ, Lederman RJ. Evidence for conduction delay in thoracic-outlet syndrome is challenged. [letter]. N Engl J Med 1984;310:1052-3.

14. Roos DB. Thoracic outlet syndrome is underdiagnosed. Muscle Nerve 1999;22:126-9 [discussion 136-7].

15. Nord KM, Kothari MJ, Kapoor P, et al. Determination of false-positive rate of thoracic outlet syndrome diagnostic maneuvers in carpal tunnel syndrome patients. [abstract]. Neurology 2005;64:A287.

16. Wright IS. The neurovascular syndrome produced by hyperabduction of the arms. Am Heart J 1945; 29:1-19.

17. Roos DB. New concepts of TOS that explain etiology, symptoms, diagnosis and treatment. Vasc Surg 1979;13:313-21.

18. Plewa MC, Delinger M. The false-positive rate of thoracic outlet syndrome shoulder maneuvers in healthy subjects. Acad Emerg Med 1998;5:337-42.
19. Remy-Jardin M, Remy J, Masson P, et al. Helical CT angiography of thoracic outlet syndrome. AJR Am J Roentgenol 2000;174:1667-74.

20. Demondion X, Bacqueville E, Paul C, et al. Thoracic outlet: assessment with $\mathrm{MR}$ imaging in asymptomatic and symptomatic populations. Radiology 2003;227:461-8.

21. Demirbag D, Unlu E, Ozdemir F, et al. The relationship between magnetic resonance imaging findings and postural maneuver and physical examination tests in patients with thoracic outlet syndrome: results of a double-blind, controlled study. Arch Phys Med Rehabil 2007;88:844-51.

22. Franklin GM, Fulton-Kehoe D, Bradley C, et al. Outcome of surgery for thoracic outlet syndrome in Washington state workers' compensation. Neurology 2000;54:1252-7.

23. Roles NC, Maudsley RH. Radial tunnel syndrome: resistant tennis elbow as a nerve entrapment. J Bone Joint Surg Br 1972;54(3):499-508.

24. van Rossum J, Buruma OJ, Kamphuisen $\mathrm{HA}$, et al. Tennis elbow-a radial tunnel syndrome? J Bone Joint Surg Br 1978;60-B(2):197-8.

25. Rosenbaum R. Disputed radial tunnel syndrome. Muscle Nerve 1999;22(7):960-7.

26. Moss SH, Switzer HE. Radial tunnel syndrome: a spectrum of clinical presentations. J Hand Surg [Am] 1983;8(4):414-20.

27. Sarhadi NS, Korday SN, Bainbridge LC. Radial tunnel syndrome: diagnosis and management. J Hand Surg [Br] 1998;23(5):617-9.

28. Dawson DM, Hallett M, Wilbourn AJ. Entrapment neuropathies. 3rd edition. Philadelphia: LippincottRaven Publishers; 1999. p. 214-9.

29. Lister GD, Belsole RB, Kleinert HE. The radial tunnel syndrome. J Hand Surg [Am] 1979;4:52-9.

30. Sotereanos DG, Varitimidis SE, Giannakopoulos PN, et al. Results of surgical treatment for radial tunnel syndrome. J Hand Surg [Am] 1999;24:566-70.

31. Jebson PJ, Engber WD. Radial tunnel syndrome: long-term results of surgical decompression. J Hand Surg [Am] 1997;22:889-96.

32. Struthers J. On a peculiarity of the humerus and humeral artery. Mon J Med Sci 1848;28:264-7.

33. Terry RJ. A study of the supracondyloid process in the living. Am J Phys Anthropol 1921;4:129-36.

34. De Jesus R, Dellon AL. Historic origin of the "Arcade of Struthers. J Hand Surg [Am] 2003; 28(3):528-31.

35. Siqueira MG, Martins RS. The controversial arcade of Struthers. Surg Neurol 2005;64(Suppl 1): S17-21.

36. Rogers MR, Bergfield TG, Aulicino PL. The failed ulnar nerve transposition. Etiology and treatment. Clin Orthop Relat Res 1991;269:193-200.

37. Kleinman WB. Revision ulnar neuroplasty. Hand Clin 1994;10:461-77. 
38. Vogel RB, Nossaman BC, Rayan GM. Revision anterior submuscular transposition of the ulnar nerve for failed subcutaneous transposition. $\mathrm{Br} \mathrm{J}$ Plast Surg 2004;57:311-6.

39. Broudy AS, Leffert RD, Smith RJ. Technical problems with ulnar nerve transposition at the elbow: findings and results of reoperation. J Hand Surg [Am] 1978; 3:85-9.

40. Caputo AE, Watson HK. Subcutaneous anterior transposition of the ulnar nerve for failed decompression of cubital tunnel syndrome. J Hand Surg [Am] 2000;25:544-51.

41. al Qattan MM, Murray KA. The arcade of Struthers: an anatomical study. J Hand Surg [Br] 1991;16(3):311-4.

42. Bartels RH, Grotenhuis JA, Kauer JM. The arcade of Struthers: an anatomical study. Acta Neurochir (Wien) 2003;145(4):295-300.

43. von Schroeder HP, Scheker LR. Redefining the "Arcade of Struthers. J Hand Surg [Am] 2003; 28(6):1018-21.

44. Wehrli L, Oberlin C. The internal brachial ligament versus the arcade of Struthers: an anatomical study. Plast Reconstr Surg 2005;115(2):471-7.

45. Spinner M. Injuries to the major branches of peripheral nerves of the forearm. 2nd edition. Philadelphia: W.B. Saunders; 1978. p. 234-7.

46. Ochiai N, Hayashi T, Ninomiya S. High ulnar nerve palsy caused by the arcade of Struthers. J Hand Surg 1992;17B:629-31.

47. Ochiai N, Honmo J, Tsujino A, et al. Electrodiagnosis in entrapment neuropathy by the arcade of Struthers. Clin Orthop Relat Res 2000;378:129-35.

48. Yuen EC, So YT. Sciatic neuropathy. Neurol Clin 1999;17:617-31.

49. Beaton LE, Anson BJ. The relationship of the sciatic nerve and of its subdivisions to the piriformis muscle. Anat Rec 1937;70:1-3.

50. Beaton LE, Anson BJ. The sciatic nerve and the piriformis muscle: Their interrelation a possible cause of coccygodynia. J Bone Joint Surg Am 1938;20:686-8.

51. Silver JK, Leadbetter WB. Piriformis syndrome: assessment of current practice and literature review. Orthopedics 1998;21(10):1133-5.

52. Stewart JD. The piriformis syndrome is overdiagnosed. Muscle Nerve 2003;28(5):644-6.

53. Fishman LM, Schaefer MP. The piriformis syndrome is underdiagnosed. Muscle Nerve 2003;28(5):646-9.

54. McCrory $P$. The "piriformis syndrome"-myth or reality? Br J Sports Med 2001;35(4):209-10.

55. Pecina M. Contribution to the etiological explanation of the piriformis syndrome. Acta Anat (Basel) 1979; 105(2):181-7.

56. Fishman LM, Zybert PA. Electrophysiologic evidence of piriformis syndrome. Arch Phys Med Rehabil 1992;73(4):359-64.

57. Pace J, Nagle D. Piriformis syndrome. West J Med 1976;124:435-9.
58. Freiberg AH. Sciatic pain and its relief by operation on muscle and fascia. Arch Surg 1937;34:337-49.

59. Beatty RA. The piriformis muscle syndrome: a simple diagnostic maneuver. Neurosurgery 1994;34(3): 512-4.

60. Fishman LM, Dombi GW, Michaelsen C, et al. Piriformis syndrome: diagnosis, treatment, and outcomea 10 year study. Arch Phys Med Rehabil 2003;83: 295-301.

61. DeLisa JA, Hang JL, Baran EM, et al. Manual of nerve conduction velocity and clinical neurophysiology. 3rd edition. Philadelphia: Lippincott Williams \& Wilkins; 1994. p. 168-79.

62. Havel PE, Ebraheim NA, Clark SE, et al. Tibial nerve branching in the tarsal tunnel. Foot Ankle 1988;9: 117-9.

63. Reade BM, Longo DC, Keller MC. Tarsal tunnel syndrome. Clin Podiatr Med Surg 2001;18(3): 395-408.

64. Stewart JD. Focal peripheral neuropathies. 1st edition. New York: Elsevier; 1987. p. 313-4.

65. Kinoshita M, Okuda R, Morikawa J, et al. The dorsiflexion-eversion test for diagnosis of tarsal tunnel syndrome. J Bone Joint Surg Am 2001;83-A(12): 1835-9.

66. Galardi G, Amadio S, Maderna L, et al. Electrophysiologic studies in tarsal tunnel syndrome. Diagnostic reliability of motor distal latency, mixed nerve and sensory nerve conduction studies. Am J Phys Med Rehabil 1994;73:193-8.

67. Oh SJ, Sarala PK, Kuba T, et al. Tarsal tunnel syndrome: electrophysiological study. Ann Neurol 1979;5(4):327-30.

68. Patel AT, Gaines K, Malamut R, et al. Usefulness of electrodiagnostic techniques in the evaluation of suspected tarsal tunnel syndrome: an evidencebased review. Muscle Nerve 2005;32:236-40.

69. Falck B, Alaranta H. Fibrillation potentials, positive sharp waves and fasciculation in the intrinsic muscles of the foot in healthy subjects. J Neurol Neurosurg Psychiatry 1983;46:681-3.

70. Gatens PF, Saeed MA. Electromyographic findings in the intrinsic muscles of normal feet. Arch Phys Med Rehabil 1982;63:317-8.

71. Dumitru D, Diaz CA, King JC. Prevalence of denervation in paraspinal and foot intrinsic musculature. Am J Phys Med Rehabil 2001;80:482-90.

72. Cimino WR. Tarsal tunnel syndrome: review of the literature. Foot Ankle 1990;11:47-52.

73. Bailie DS, Kelikian AS. Tarsal tunnel syndrome: diagnosis, surgical technique, and functional outcome. Foot Ankle Int 1998;19(2):65-72.

74. Wood WA, Wood MA. Decompression of peripheral nerves for diabetic neuropathy in the lower extremity. $\mathrm{J}$ foot Ankle Surg 2003;42:268-75.

75. Dellon AL. Neurosurgical prevention of ulceration and amputation by decompression of lower 
extremity peripheral nerves in diabetic neuropathy: update 2006. Acta neurochir Suppl 2007;100: 149-51.

76. Ducic I, Taylor NS, Dellon AL. Relationship between peripheral nerve decompression and gain of pedal sensibility and balance in patients with peripheral neuropathy. Ann Plast Surg 2006;56:145-50.

77. Caffee $\mathrm{HH}$. Treatment of diabetic neuropathy by decompression of the posterior tibial nerve. Plast Reconstr Surg 2000;106:813-5.

78. Aszmann OC, Kress KM, Dellon AL. Results of decompression of peripheral nerves in diabetics: a prospective blinded study. Plast Reconstr Surg 2000;106:816-22.

79. Dellon AL. Treatment of symptomatic diabetic neuropathy by surgical decompression of multiple peripheral nerves. Plast Reconstr Surg 1992;89: 689-97.

80. Chaudhry V, Stevens JC, Kincaid J, et al. Practice advisory: utility of surgical decompression for treatment of diabetic neuropathy. Report of the Therapeutics and Technology Assessment Subcommittee of the American Academy of Neurology. Neurology 2006;66:1805-8. 